PAPER

\title{
Effects of botulinum toxin type $A$ on vibration induced facilitation of motor evoked potentials in spasmodic torticollis
}

\section{P P Urban, R Rolke}

See end of article for authors' affiliations

.....................

Correspondence to: Dr P D P P Urban, Department of Neurology, University of Mainz, Langenbeckstr. 1, D 55101 Mainz; urban@neurologie.klinik. uni-mainz.de

Received

26 September 2003

In revised form

14 January 2004

Accepted 5 February 2004

\begin{abstract}
Background and aim: It has not been clarified if botulinum toxin (BTX) injection leads to muscle spindle dysfunction in man. This study aimed to test the hypothesis that BTX application reduces the facilitation of a magnetic evoked response (MEP).

Methods: We used the vibration induced facilitation of an MEP of the sternocleidomastoid muscle (SCM) as a surrogate marker for muscle spindle function in 20 healthy subjects and 10 patients with idiopathic rotational torticollis in whom BTX was injected unilaterally.

Results: The increase in the amplitude and area of the MEPs in the clinically not affected and untreated SCM of the patients did not differ significantly from the controls. At baseline, the vibration induced increase in the affected SCM of the patients was significantly lower than in the control group. Six weeks after BTX application, the observed facilitation decreased significantly (amplitude and area: $p<0.001$ ) when compared with baseline values. Twelve weeks after BTX application, facilitation showed an increase in the values of the amplitude $(p<0.001)$, but not of the area when compared with the six week examination.

Conclusions: We demonstrated that the vibration induced MEP facilitation in the SCM of patients with torticollis significantly decreased six weeks after BTX application and again increased after 12 weeks in part when looking at MEP amplitude. This observation is suggestive of denervation and reinnervation of the muscle spindles after BTX injection.
\end{abstract}

dystonic active sternocleidomastoid muscle (SCM) was injected unilaterally (left SCM, $\mathrm{n}=7$; right SCM, $\mathrm{n}=3$ ) in the upper third of the muscle directly after baseline measurement and was designated as "affected SCM". The SCM which was not responsible for the head rotation and which was not treated was designated as "not affected $\mathrm{SCM}^{\prime}$. The patients were treated with either Dysport $(\mathrm{n}=5$, dosage ranging from $100 \mathrm{U}$ to $300 \mathrm{U}$; Ipsen Pharma, Ettlingen, Germany) or Botox $(\mathrm{n}=5$, dosage ranging from $20 \mathrm{U}$ to $60 \mathrm{U}$; Merz Pharmaceuticals, Frankfurt, Germany).

The patients were examined in a supine position and the head facing straight upwards. The contralateral motor cortex was magnetically stimulated at an intensity 30\% above the motor threshold at rest, using a circular coil (diameter $9 \mathrm{~cm}$, Magstim 200; Magstim Co, London, UK) positioned tangentially 3-4 cm lateral to the vertex. The threshold was defined as the minimal intensity of stimulator output, eliciting three responses of at least $50 \mu \mathrm{V}$ peak-to-peak amplitude for five stimuli. MEPs were recorded from the relaxed SCM using surface electrodes (no. 838546; Hellige, Freiburg, Germany) in a belly tendon arrangement with the active electrode placed on the middle of the upper half of the SCM, and the inactive electrode placed on the mastoid. Relaxation of the SCM was achieved by feedback using continuous electromyographic monitoring. The lower half of the SCM was vibrated with a pneumatic vibrator powered by compressed air at a frequency of $85 \mathrm{~Hz}$ (Model 2Z431C; Dayton Electric Co, Chicago, IL, USA). Five MEPs before and three seconds after the onset of vibration of the SCM were averaged. The peak-to-peak amplitude and the area of the average curve

Abbreviations: ANOVA, analysis of variance; BTX, botulinum toxin; MEP, motor evoked potential; SCM, sternocleidomastoid muscle; TMS, transcranial magnetic stimulation using the Tsui scale. ${ }^{9}$ None of the patients received additional oral drugs during the study period. In all patients, the 
were measured, and the relative differences without and with vibration were calculated (\%). Logarithmic transformation of MEP data prior to checking the assumption of normality indicated that logarithmically transformed values were normally distributed (fig 1A, B).

Statistical analysis was performed (log transformed data with base 10) using Statistica software, release 5.1 (StatSoft Inc, Tulsa, OK, USA). Analysis of variance (ANOVA) for repeated measures was calculated with LSD-post hoc tests. The study was approved by the local ethics committee (State Medical Council Rhineland-Palatinate, No. 3312), and informed consent was obtained from all subjects.

\section{RESULTS}

In healthy volunteers, there were no significant differences (paired $t$ tests, $\mathrm{p}=0.998$ ) between the measurements of the left and right SCM (for an example see fig 2A, B). The mean vibration induced increase of amplitude was 262\% (95\% confidence interval (CI) of mean $111 \%$ to $619 \%)$, while the area of the test response increased by $238 \%$ (95\% CI of mean $100 \%$ to $567 \%$ ). The large range of the $95 \%$ confidence intervals of means indicate that the observation of almost missing (amplitude) or completely missing (area) vibration induced facilitation of MEP response in SCM is within the normal range due to the large variability of measurements.

The latency and stimulation threshold of the healthy volunteers and the patients over the unaffected SCM (for example see fig 2) did not differ significantly. Comparison of
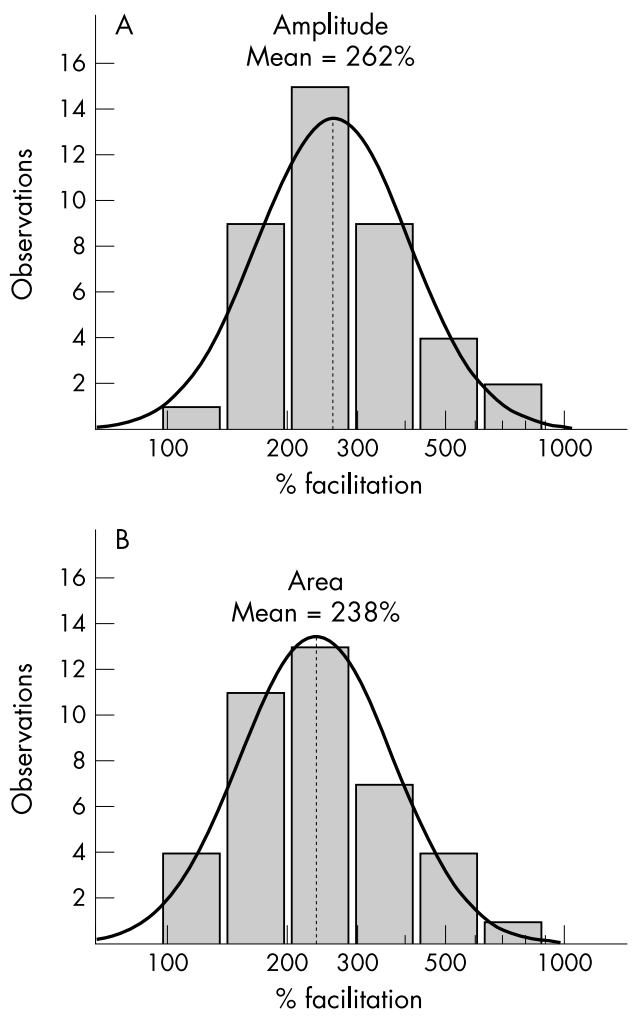

Figure 1 (A) Distribution of vibration induced facilitation of MEP amplitude (\%). Normative data of MEP amplitude after facilitation were normally distributed after log transformation (Kolmogorov-Smirnov's $\mathrm{D}=0.06, \mathrm{p}=$ not significant (ns)) with a mean facilitation of 262 $\left(=10^{2.419 \pm 0.190}\right) \%(n=40)$. Note the logarithmic scale on the $x$-axis. (B) Distribution of vibration induced facilitation of MEP response (area under the curve; $\mathrm{mV}$ expressed as facilitation in \%). Normative data of MEP response after facilitation were normally distributed after log transformation (Kolmogorov-Smirnov's $D=0.05, p=n s$ ) with a mean facilitation of $238\left(=10^{2.377 \pm 0.192}\right) \%(n=40)$. Note the logarithmic scale on the $x$-axis. the controls and unaffected SCM of the patients showed there were no significant differences with regard to MEP amplitude and area at any time interval $\left(\mathrm{F}_{3,27}=0.33\right)$. In patients with spasmodic torticollis, the increase in the amplitude (mean 276\%) and area (mean 250\%) of the MEPs in the clinically not affected and untreated SCM did not differ significantly from controls. To quantitatively assess atrophy of the affected SCM, we compared the baseline values of affected and unaffected SCM. MEP responses did not differ significantly regarding amplitude (paired $t$ test, $\mathrm{p}=0.16$ ) and area (paired $t$ test, $\mathrm{p}=0.79$ ), indicating that at the time of baseline measurement no relevant atrophy of the affected SCMs was detected (tables 1 and 2). The vibration induced increase in the patients' affected SCM was significantly lower than in the controls (amplitude and area: LSD-post hoc test, $\mathrm{p}<0.001$ ) as well as in their not affected SCM (amplitude and area: LSD-post hoc test, $\mathrm{p}<0.001$ ).

Significant differences were detected when comparing affected SCM at baseline, six, and 12 weeks $\left(F_{2,18}=14.1\right)$. Six weeks after BTX application, the observed facilitation decreased significantly (amplitude and area: LSD-post hoc test, $\mathrm{p}<0.001$ ) compared with baseline values (fig 3A). Twelve weeks after BTX application, facilitation was increased for the amplitude (LSD-post hoc test, $\mathrm{p}<0.001$ ) but not for the area values (LSD-post hoc test, $p=n s$ ) compared with the six week examination (fig 3A). Moreover, we calculated six weeks versus baseline ratios of amplitude and area of MEP responses with and without vibration. Mean six weeks/baseline ratio of MEP amplitude with vibration was 0.72 , and without vibration was 0.95 . Mean six weeks/baseline ratio of MEP area with vibration was 0.52 , and without vibration was 0.70 . The differences between the ratios were clearly significant (paired $t$ test; $\mathrm{p}<0.01$ for amplitude and area). These data show 23\% (amplitude) and 18\% (area) more decrease of MEP response after BTX with vibration than without vibration (raw MEP response), indicating that the decrease of vibration induced facilitation of MEP response is not only mediated by extrafusal muscle fibres.

The clinical improvement after BTX application is reflected by the Tsui score. The mean Tsui score at baseline was 10.3 (SD 2.3) and decreased six weeks after BTX treatment to 5.2 (1.9) and increased again 12 weeks after BTX to 9.6 (2.1). ANOVA revealed highly significant differences over time $\left(\mathrm{F}_{2,18}=104\right)$. Looking at clinical ratings over time, Tsui score at baseline and 12 weeks showed no significant differences, while the score six weeks after BTX therapy compared with baseline and 12 weeks was highly significantly reduced (LSD-post hoc test, $\mathrm{p}<0.001$ ).

Although head rotation was not assessed quantitatively, vibration itself had no clinical influence on the spasmodic torticollis.

\section{DISCUSSION}

It has been demonstrated in a rat model that BTX is associated with intrafusal muscle fibre atrophy ${ }^{2}$ and with a reduction in the spindle afferent (Ia) discharge rate from jaw muscle spindles. ${ }^{3}$ Furthermore, intramuscularly applied lidocaine, which blocks Ia afferences and $\gamma$-efferences, ${ }^{56}$ improves dystonic movement in patients with writer's cramp ${ }^{4}$ and oromandibular dystonia. ${ }^{10}$ In patients with blepharospasm, however, lidocaine and alcohol showed no ${ }^{10}$ or a very mild and transient effect. ${ }^{11}$ The conflicting reports of the efficacy of lidocaine might be due to the fact that the human hand muscles (abductor pollicis brevis muscle, 12.9 spindles/g) and jaw muscles (masseter muscle, 11.2 spindles/g) are richly supplied with muscle spindles, ${ }^{12}{ }^{13}$ while the orbicularis oculi muscles have only few or no muscle spindles. ${ }^{14} 15$ However, the available data are based on only a small number of autopsies and, as demonstrated by animal studies, 

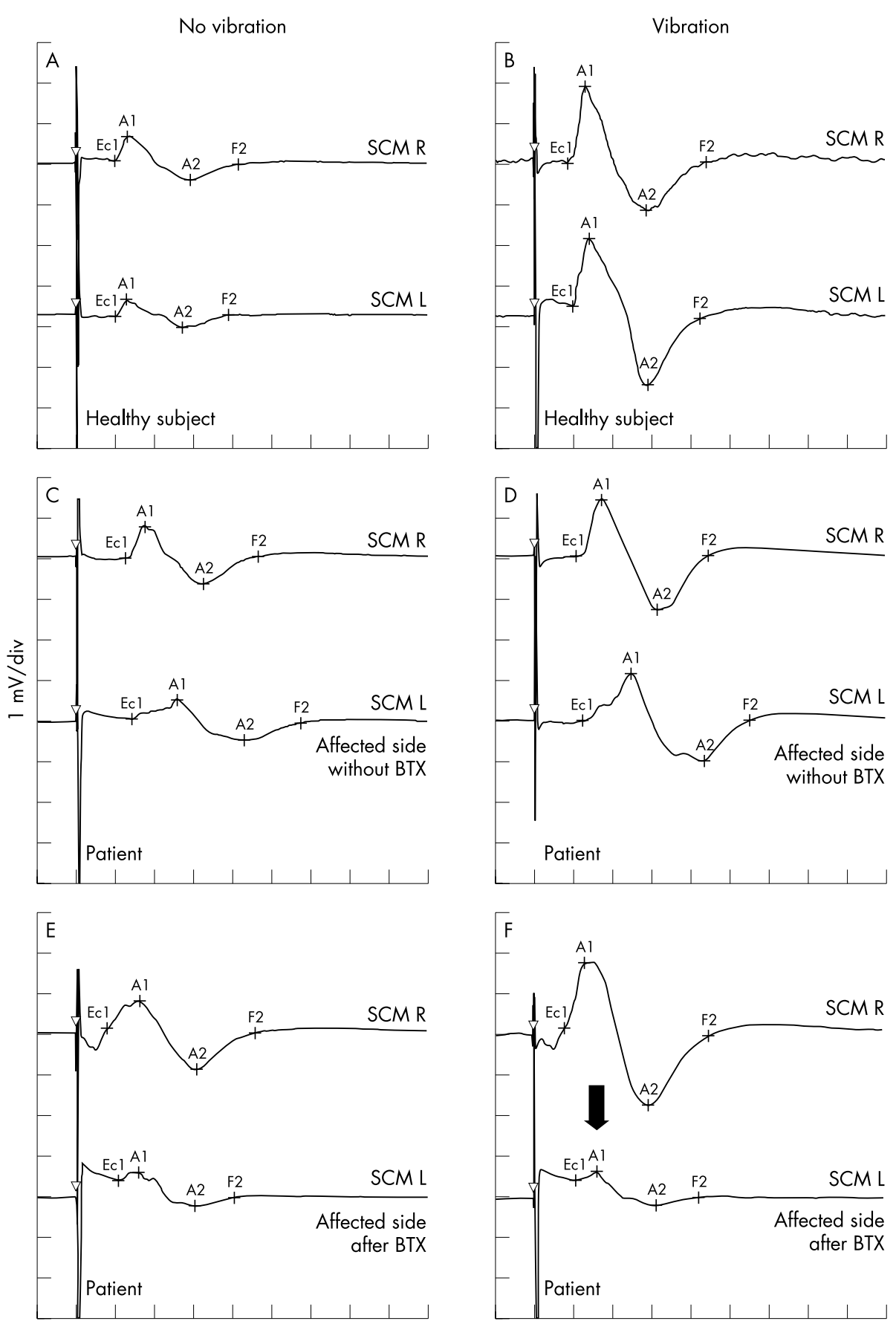

Figure 2 MEP responses with and without vibration in a healthy subject and a patient with torticollis spasmodicus. (A, C, E) Raw MEP responses without vibration. $(B, D, F)$

MEP responses with vibration. Average response of the sternocleidomastoid muscle (SCM) to five transcranial magnetic stimuli of the contralateral motor cortex before $(A)$ and during vibration of the SCM (B) in a healthy volunteer. The altered background activity on the right SCM during vibration is due to a vibration induced electrode movement artefact. Average response of the SCM to five transcranial magnetic stimuli of the contralateral motor cortex before (C) and during vibration of the SCM (D) in a patient with spasmodic torticollis without botulinum toxin (BTX) treatment. Six weeks after BTX treatment of the left $S C M$ the raw MEP response without vibration is reduced (E). During vibration (F) the MEP response still remains reduced (black arrow), while the untreated SCM shows facilitation. $R$, right; L, left.

transduction in sensory nerve fibres. ${ }^{21}$ We therefore used the facilitation factor between before and after vibration MEPs as a surrogate for muscle spindle function and Ia afferent input. This approach is supported by the observation that, in contrast with the SCMs, only minor facilitation (median facilitation, $130 \%$ in 13 healthy subjects; own unpublished observations) of the MEP is observed in facial muscles, which have only few or no muscle spindles. The recordings were performed from the buccinator muscle with a special device adapted to the oral vestibulum, ${ }^{22}$ where the electrode surfaces were in contact with the inner side of the cheek and vibration was applied to the outer side of the cheek.

On the basis of the described observations we hypothesised that BTX also denervates intrafusal muscle fibres in man, and 
Table 1 Amplitudes $(\mathrm{mV})$ of the compound action potentials of the sternocleidomastoid muscle (SCM) before botulinum toxin (BTX) treatment (baseline), and six and 12 weeks after BTX treatment of the unaffected and affected SCM following transcranial magnetic stimulation of the motor cortex

\begin{tabular}{|c|c|c|c|c|c|c|c|c|c|c|c|c|}
\hline \multicolumn{4}{|c|}{ Baseline } & \multicolumn{4}{|c|}{6 weeks } & \multicolumn{4}{|c|}{12 weeks } & \\
\hline \multicolumn{2}{|c|}{ Not affected SCM } & \multicolumn{2}{|c|}{ Affected SCM } & \multicolumn{2}{|c|}{ Not affected SCM } & \multicolumn{2}{|c|}{ Affected SCM } & \multicolumn{2}{|c|}{ Not affected SCM } & \multicolumn{2}{|c|}{ Affected SCM } & \\
\hline Base & Vibration & Base & Vibration & Base & Vibration & Base & Vibration & Base & Vibration & Base & Vibration & \\
\hline 3.4 & 7 & 1.6 & 1.9 & 1.4 & 2.5 & 0.8 & 0.8 & 2.6 & 6.5 & 1.4 & 1.7 & \\
\hline 0.5 & 1.7 & 1.5 & 1.9 & 0.3 & 0.9 & 1.2 & 1.3 & 0.7 & 1.8 & 1.3 & 1.6 & \\
\hline 0.8 & 2.3 & 0.9 & 1.6 & 1.1 & 2.9 & 0.3 & 0.3 & 0.8 & 2 & 0.7 & 0.9 & \\
\hline 0.4 & 1.1 & 0.3 & 0.5 & 0.5 & 1.5 & 0.5 & 0.6 & 0.4 & 0.9 & 0.3 & 0.5 & \\
\hline 0.3 & 0.7 & 1.6 & 2.3 & 0.4 & 1 & 2.1 & 2.5 & 1.3 & 3.4 & 1.9 & 2.5 & \\
\hline 0.7 & 3.8 & 2 & 2.7 & 0.5 & 1.4 & 2.2 & 2.5 & 1.9 & 3.4 & 2.3 & 3.1 & \\
\hline 1.2 & 2.3 & 5.7 & 5.9 & 1.4 & 2.9 & 4.6 & 4 & 2.5 & 5.7 & 5.4 & 5.2 & \\
\hline 0.8 & 1.7 & 3.2 & 3.6 & 1.2 & 1.9 & 0.6 & 0.5 & 2.4 & 4.1 & 2.7 & 3 & \\
\hline 0.7 & 4.1 & 0.4 & 1 & 0.8 & 2.8 & 0.6 & 0.9 & 0.7 & 2.8 & 0.6 & 0.7 & \\
\hline 0.3 & 0.8 & 0.3 & 0.8 & 0.4 & 1.4 & 0.4 & 0.8 & 0.5 & 1.6 & 0.4 & 0.8 & \\
\hline 0.91 & 2.55 & 1.75 & 2.22 & 0.8 & 1.92 & 1.33 & 1.42 & 1.38 & 3.22 & 1.7 & 2 & (Mean) \\
\hline
\end{tabular}

Base, baseline measurement

therefore reduces facilitation of the MEPs in the vibrationTMS paradigm. To test this hypothesis, we selected patients with idiopathic rotational torticollis who were treated with BTX depending on the clinical indication. The SCM was selected as the target muscle for the vibration-TMS paradigm, because this muscle is large enough for both the application of vibration stimuli (in the lower half) and simultaneous recording of electromyographic activity using surface electrodes (in the upper half). This was the reason why the investigations were performed in patients and not in healthy volunteers where only relatively small muscles-for example, the extensor digitorum brevis muscle, can be treated with BTX without relevant functional deficits. In the SCM, morphometric studies have demonstrated a muscle spindle density of 5.7 spindles/g. ${ }^{12}$ Thus, the occurrence of vibratory effects was assumed. TMS of the contralateral motor cortex readily evoked muscle potentials in the SCM, despite the fact that the muscle receives bilateral projections from the motor cortex. $^{23-25}$

The results of our study demonstrate that the vibration induced facilitation in the treated SCM of patients with spasmodic torticollis is significantly lower six weeks after BTX application compared with the values recorded at baseline on the untreated side as well as in healthy volunteers. This observation might speak in favour of denervation of the muscle spindles as observed in cat and rat models showing that BTX is associated with intrafusal muscle fibre atrophy ${ }^{2}$ and reduction in the spindle afferent (Ia) discharge rate from jaw muscle spindles. ${ }^{3}$ Evidence for reduced Ia afferences after BTX treatment in man is sparse. After BTX treatment of patients with upper limb dystonia, Priori et $a^{26}$ observed changes in the reciprocal inhibition between forearm muscles and Gilio et $a l^{27}$ described a transient reduction of intracortical inhibition. Both phenomena were assumed to be mediated by an altered muscle spindle input.

However, reduced or absent facilitation of MEPs by vibration may also be observed in voluntarily activated muscles. An explanation of this phenomenon may be that the muscle spindles are already fully active during voluntary contraction due to $\alpha-\gamma$ coactivation. ${ }^{28}$ However, the reduced facilitation in our patients can not be explained by greater (dystonic) muscle activation, because the absence of electromyographic activity during TMS was checked by online monitoring. Most unlikely the reduced MEP facilitation can be attributed to the dystonic activity of the SCM, because this reduction in facilitation was greatest when BTX was clinically most effective (six weeks after BTX), as demonstrated by a lower dystonic activity score (Tsui score) compared with that at baseline. Thus, the reduced MEP facilitation is most probably associated with the BTX treatment. In view of the fact that no patient had relevant atrophy of the muscle as explained before, and that the absolute amplitude of the MEPs was still large enough to show facilitation, it is unlikely

Table 2 Area under the curve $(\mathrm{mVms})$ of the compound action potentials of the sternocleidomastoid muscle (SCM) before BTX treatment (baseline), and six and 12 weeks after BTX treatment of the unaffected and affected SCM following transcranial magnetic stimulation of the motor cortex

\begin{tabular}{|c|c|c|c|c|c|c|c|c|c|c|c|c|}
\hline \multicolumn{4}{|c|}{ Baseline } & \multicolumn{4}{|c|}{6 weeks } & \multicolumn{4}{|c|}{12 weeks } & \\
\hline \multicolumn{2}{|c|}{ Not affected SCM } & \multicolumn{2}{|c|}{ Affected SCM } & \multicolumn{2}{|c|}{ Not affected SCM } & \multicolumn{2}{|c|}{ Affected SCM } & \multicolumn{2}{|c|}{ Not affected SCM } & \multicolumn{2}{|c|}{ Affected SCM } & \\
\hline Base & Vibration & Base & Vibration & Base & Vibration & Base & Vibration & Base & Vibration & Base & Vibration & \\
\hline 11.8 & 25.2 & 3.7 & 4.4 & 9.2 & 21.6 & 3.1 & 2.6 & 10.2 & 23.3 & 3.9 & 3.3 & \\
\hline 6.1 & 10.9 & 13 & 15.6 & 6.3 & 9.8 & 10.3 & 10 & 7.8 & 14.9 & 11 & 10.8 & \\
\hline 6.7 & 21.2 & 8.7 & 16.9 & 8.7 & 26 & 1.1 & 1.7 & 8.2 & 22.9 & 6.3 & 9.7 & \\
\hline 3.1 & 8.5 & 5.8 & 11.4 & 4.2 & 11.9 & 5.1 & 5.2 & 2.4 & 6.5 & 6.2 & 6.3 & \\
\hline 16.9 & 30.9 & 3.5 & 4.9 & 11.3 & 18.9 & 2.7 & 3.3 & 22 & 42.7 & 6.5 & 7.9 & \\
\hline 15.4 & 31.7 & 13.6 & 19.6 & 16.7 & 37.1 & 11.8 & 14.4 & 13.5 & 26.6 & 17.3 & 21.1 & \\
\hline 31.9 & 49.4 & 8.5 & 8.8 & 55 & 92.9 & 6.1 & 5.4 & 46.6 & 69.4 & 13.2 & 11.7 & \\
\hline 8.2 & 16.8 & 29.9 & 36.5 & 7.7 & 18.8 & 9.1 & 8.9 & 8.3 & 19.5 & 18.5 & 18.1 & \\
\hline 6.8 & 38.4 & 8.3 & 13.1 & 5.6 & 27.2 & 7.6 & 8.6 & 5.2 & 27.8 & 8.1 & 9.2 & \\
\hline 1 & 4.2 & 2.3 & 5.4 & 0.5 & 2.2 & 1.9 & 2.7 & 0.9 & 3.7 & 2.4 & 3.4 & \\
\hline 10.8 & 23.7 & 9.7 & 13.7 & 12.5 & 26.6 & 5.9 & 6.3 & 12.5 & 25.7 & 9.3 & 10.2 & (Mean) \\
\hline
\end{tabular}



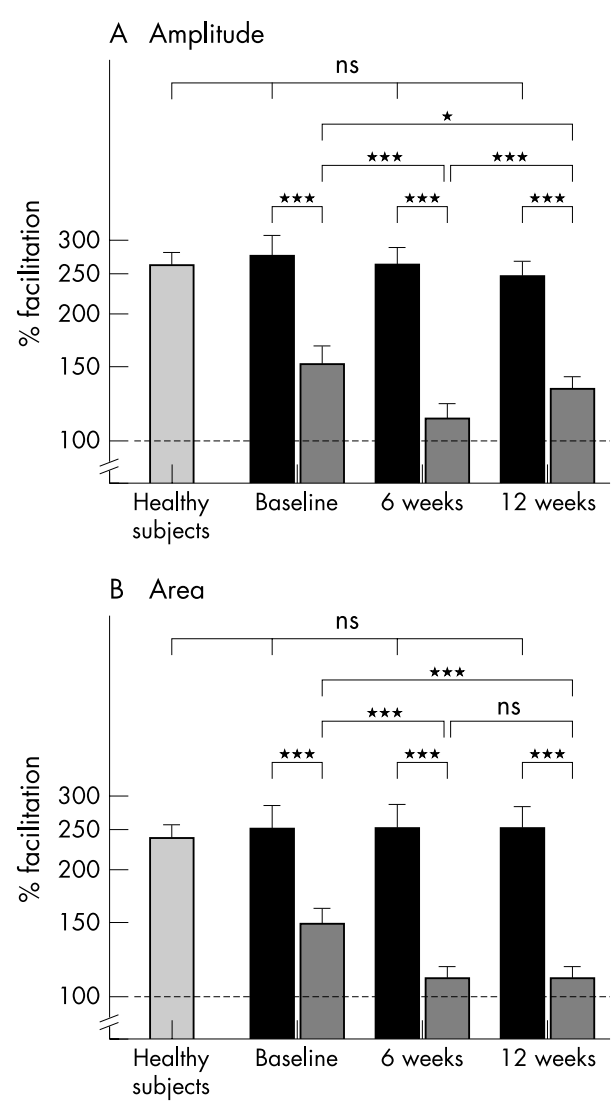

Figure 3 (A, B) Vibration induced facilitation of MEP responses. Relative increase $(\%)$ in the amplitude (A) and area (B) of the magnetic evoked potentials during vibration of both SCM in 20 healthy volunteers (light grey bar; $n=40)$, and in patients with spasmodic torticollis $(n=10)$ at baseline, and six and 12 weeks after BTX treatment (black bar, not affected SCM; dark grey bar, affected SCM). Bars represent mean (SEM). Asterisks denote the level of significance (LSD, post-hoc comparison, $\left.{ }^{*} p<0.05\right)$. Mean vibration induced facilitation of MEP responses did not differ significantly between healthy subjects and unaffected SCM at any time. Affected and unaffected SCM showed highly significant differences (LSD, post-hoc comparison, ${ }^{* * *} p<0.001$ ) at each corresponding day of investigation. Note the logarithmic scale on the $y$-axis.

that the reduced facilitation is due to the effects of BTX on the extrafusal fibres only. The possibility that the paresis of extrafusal fibres could lead to "slack" intrafusal fibres that make Ia afferents less responsive to vibration ${ }^{29}$ seems also unlikely due to the same experimental conditions before and after BTX treatment - that is, the muscle was examined in a relaxed state and the vibration device was tightly pressed against the underlying muscle leading to a passive muscle stretch.

Twelve weeks after BTX, the facilitation of the MEP amplitude increased significantly compared with the six week examination, whereas the MEP area only showed a similar facilitation. This observation might speak in favour of reinnervation of the muscle spindles observed in cat and rat models after peripheral nerve lesions. ${ }^{30} 31$ Serial electron microscopic studies, however, demonstrated that motor reinnervation of the muscle spindles occurred following nerve transsection in the majority of patients after an interval of six months or later. ${ }^{32}$ Even though the increase of MEP amplitude at 12 weeks interval (almost returning to baseline) was significant when compared with six weeks, there was no remarkable increase of MEP area, indicating that these different measures possibly reflect the differences in functional reorganisation in terms of reinnervation of extrafusal and intrafusal muscle fibres. The fact that there was no increase after 12 weeks in the MEP area of our patients might predominantly be explained by delayed reinnervation process of intrafusal fibres, which seem to play an important role in vibration induced facilitation of MEP response. It may therefore be speculated that on re-examination after six months and later a further increase in facilitation could be observed. However, the patients with torticollis deteriorated clinically after three months. Thus, it would not have been justified to delay the next BTX application to answer this question.

The finding that the baseline measurements of patients with ongoing BTX treatment showed significantly less facilitation compared with both the controls and the unaffected SCM might be due to a carry-over effect from previous BTX applications. The pretreatment facilitation in the de novo patients did not differ from that of controls. However, the pretreatment facilitation in the de novo patients was also lower than on the unaffected side of the patients with spasmodic torticollis. For this observation two explanations are possible. First, the deficient facilitation before BTX might be a consequence of preactivation of $\gamma$-efferents that make group Ia afferents busy in the resting state (see below). Secondly, the reduced facilitation before BTX might support the assumption of the presence of abnormal central sensorimotor processing of the proprioceptive input. This view is also supported by an abnormal perception of vibration induced illusion of movement in focal dystonia $^{33}$ and by a decrease in activation of the primary sensorimotor and supplementary motor area (SMA) in patients with writer's cramp and blepharospasm observed by $\mathrm{H}_{2}{ }^{15} \mathrm{O}$-PET (positron emission tomography) investigations during vibration of the affected muscles. ${ }^{34} 35$ However, in another $\mathrm{H}_{2}{ }^{15} \mathrm{O}$-PET study, BTX treatment failed to improve impaired activation of the primary motor cortex during volitional movement in patients with idiopathic torsion dystonia. ${ }^{36}$ While these findings may be interpreted as a consequence of either a change in movement strategy, or as cortical reorganisation secondary to de-efferentation of $\alpha$-motor neurones, they do not serve as evidence of an altered afferent input following BTX treatment.

Observation of the clinical effects of vibration on dystonic muscles yielded inconsistent results. Vibration itself was reported not only to provoke dystonic movement in writer's cramp, ${ }^{47}$ but also to improve the symptoms of spasmodic torticollis.$^{38}$ In our patients, vibration did not exert an effect on cervical dystonia.

We conclude that in accordance with previous animal studies, our findings in man suggest that BTX not only denervates extrafusal fibres as reflected by the absolute amplitude reduction following TMS, but also denervates intrafusal fibres with a prolonged reduction of vibration induced MEP area. It may therefore be justified to assume that a reduced primary muscle spindle input after BTX application contributes to the clinical efficacy of the treatment for spasmodic torticollis.

\section{ACKNOWLEDGEMENTS}

We wish to thank Ms I Kirchhoff for her expert technical assistance and Prof Dr W A Nix for providing the vibration device.

\section{Authors' affiliations}

P P Urban, R Rolke, Department of Neurology, University of Mainz, Germany

R Rolke, Institute of Physiology and Pathophysiology, University of Mainz, Germany

This work was supported by BMBF grant 01 EM0107 (German Research Network on Neuropathic Pain, GNNP).

Competing interests: none declared 


\section{REFERENCES}

1 Hallett M, Glocker FX, Deuschl G. Mechanism of action of botulinum toxin. Ann Neurol 1994;36:449.

2 Rosales RL, Arimura K, Takenaga S, et al. Extrafusal and intrafusal muscle effects in experimental botulinum toxin-A injection. Muscle Nerve 1996; 19:488-96.

3 Filippi GM, Errico P, Santarelli R, et al. Botulinum A toxin effects on rat jaw muscle spindles. Acta Otolaryngol 1993;113:400-4.

4 Kaji R, Rothwell JC, Katayama M, et al. Tonic vibration reflex and muscle afferent block in writer's cramp. Ann Neurol 1995;38:155-62.

5 Matthews $\mathbf{P}$, Rushworth $\mathrm{G}$. The relative sensitivity of muscle nerve fibres to procaine. J Physiol 1957a;135:263-9.

6 Mathews P, Rushworth $G$. The selective effect of procain on the stretch reflex and tendon jerk of soleus muscle when applied to its nerve. J Physiol 1957b; 135:245-62.

7 Roll JP, Vedel JP, Ribot E. Alteration of proprioceptive messages induced by tendon vibration in man: a microneurographic study. Exp Brain Res 1989:76:213-22

8 Claus D, Mills KR, Murray NMF. Facilitation of muscle responses to magnetic brain stimulation by mechanical stimuli in man. Exp Brain Res 1988;71:273-8.

9 Tsui JKC, Eisen A, Stoessl AJ, et al. Double-blind study of botulinum toxin in spasmodic torticollis. Lancet 1986;2:245-6.

10 Yoshida K, Kaji R, Kubori T, et al. Muscle afferent block for the treatment of oromandibular dystonia. Mov Disord 1998;13:699-705.

11 Mubaidin AF. Alcohol with xylocaine for treatment of eyelid dystonia. Eur J Neurol 2000;7:213-15.

12 Voss H. Tabelle der absoluten und relativen Muskelspindelzahlen der menschlichen Skelettmuskulatur. Anat Anz 1971;129:562-72.

13 Kubota K, Masegi T. Muscle spindle supply to the human jaw muscle. J Dent Res 1977;56:901-9.

14 Hosokawa H. Proprioceptive innervation of striated muscles in the territory of the cranial nerves. Tex Rep Biol Med 1961;19:405-64.

15 Goodmurphy CW, Oralle WK. Morphological study of two human facial muscles: orbicularis oculi and corrugator supercilii. Clin Anat 1999:12:1-11.

16 Chin NK, Cope M, Pang M. Number and distribution of spindle capsules in seven hindlimb muscles of the cat. In: Barker D, ed. Symposium on muscles receptors. Hong Kong: University Press, 1962:241-8.

17 Burke D, Hagbarth KE, Lofstedt L, et al. The responses of human muscle spindle endings to vibration on noncontracting muscles. J Physiol 1976;261:673-93.

18 Burke D, Hagbarth KE, Lofstedt L, et al. The responses of human muscle spindle endings to vibration during isometric contraction. J Physiol 1976:261:695-711.

19 Roll JP, Vedel JP. Kinaesthetic role of muscle afferents in man, studied by tendon vibration and microneurography. Exp Brain Res 1982;47:177-90.
20 Kossev A, Siggelkow S, Schubert $M$, et al. Muscle vibration: different effects on transcranial magnetic and electrical stimulation. Muscle Nerve 1999;22:946-8.

21 Kessler KR, Benecke R. Botulinum toxin: from poison to remedy. Neurotoxicology 1997;18:761-70.

22 Urban PP, Beer S, Hopf HC. Cortico-bulbar fibres to orofacial muscles: recordings with enoral surface electrodes. Electroenceph Clin Neurophysiol 1997; 105:8-14.

23 Gandevia SC, Applegate $C$. Activation of neck muscles from the human motor cortex. Brain 1988;111:801-13.

24 Thompson ML, Thickbroom GW, Mastaglia FL. Corticomotor representation of the sternocleidomastoid muscle. Brain 1997; 120:245-55.

25 DeToledo JC, Dow R. Sternomastoid function during hemispheric suppression by Amytal: insights into the inputs to the spinal accessory nerve nucleus. Mov Disord 1998;13:809-12.

26 Priori A, Berardelli A, Mercuri B, et al. Physiological effects produced by botulinum toxin treatment of upper limb dystonia. Brain 1995;118:801-7.

27 Gilio F, Curra A, Lorenzano C, et al. Effects of botulinum toxin type A on intracortical inhibition in patients with dystonia. Ann Neurol 2000;48:20-6

28 Vallbo AB, Hagbarth KE, Torebjörk, et al. Somatosensory, proprioceptive, and sympathetic activity in human peripheral nerves. Physiol Rev 1979;59:919-57.

29 Gregory JE, Wise AK, Wood SA, et al. Muscle history, fusimotor activity and the human stretch reflex. J Physiol 1998;513:927-34

30 Brown MC, Butler RG. Regeneration of afferent and efferent fibres to muscle spindles after nerve injury in adult cats. J Physiol 1976;260:253-66.

31 Hyde D, Scott JJA. Responses of cat peroneus brevis muscle spindle afferents during recovery from nerve-crush injury. J Neurophysiol 1983;50:344-57.

32 Dieler R, Völker A, Schröder JM. Scanning electron microscopic study of denervated and reinnervated intrafusal muscle fibres in rats. Muscle Nerve 1992; 15:433-41.

33 Rome S, Grünewald RA. Abnormal perception of vibration induced illusion of movement in dystonia. Neurology 1999;53:1794-800

34 Tempel LW, Perlmutter JS. Abnormal cortical responses in patients with writer's cramp. Neurology 1993;43:2252-7.

35 Feiwell RJ, Black KJ, McGee-Minnich LA, et al. Diminished regional cerebral blood flow response to vibration in patients with blepharospasm. Neurology 1999:52:291-7.

36 Ceballos-Baumann AO, Sheean G, et al. Botulinum toxin does not reverse the cortical dysfunction associated with writer's cramp. Brain 1997;120:571-82.

37 Tempel LW, Perlmutter JS. Abnormal vibration-induced cerebral blood-flow responses in idiopathic dystonia. Brain 1990;113:691-707.

38 Leis AA, Dimitrijevic MR, Delapasse JS, et al. Modification of cervical dystonia by selective sensory stimulation. J Neurol Sci 1992;1 10:79-89. 